\title{
Double Interfacial Layers Effect on Optical Third-order Nonlinear Susceptibility, Refraction Index, and Absorption Coefficient of a Metal/Dielectric Composite
}

\author{
Berhanu Aragie \\ Department of Physics, CNCS, Wolkite University, P.O.Box 007, Wolkite, Ethiopia
}

Copyright (C)2019 by authors, all rights reserved. Authors agree that this article remains permanently open access under the terms of the Creative Commons Attribution License 4.0 International License

\begin{abstract}
We investigate the way of enhancing the optical third order susceptibility, the refractive index, and absorption coefficient of a composite media in which identical nonlinear nanospheres having double interfacial layer randomly embedded in the linear host medium. We observe two maxima peaks of the nonlinear properties. We also show that the effect of double interfacial layers on the third order susceptibility, the refractive index, and absorption coefficient depends on the volume fraction metal/dielectric nanosphers and the nature of the double interfacial layers. Under appropriate condition (nature of the two interfacial layer) we found two maximum peaks of the nonlinear properties. We also compare with the same composite without interfacial layer and in the presence of single interfacial layer and our finding shows that because of additional interfacial layer the effective medium exhibit a better third-order susceptibility, refractive index, and absorption coefficient.
\end{abstract}

Keywords Double Interfacial Layers, Third Order Susceptibility; Refractive Index; Absorption Coefficient

\section{Introduction}

The physics of nonlinear optical property of a disordered metal/dielectric composites becomes the most scientifically significant area in the developments of modern optical devices. Because of its potential, now a day, it received a great attention in the area of memory element, optical transistor, and all-optical switching [1, 2, 3].

For a system composed of a metal/dielectric nanospheres randomly embedded in a linear (or nonlinear) dielectric host, studding the effective nonlinear optical response is an important point of nonlinear optics. Optical bistability [4] and optical percolation threshold [5] are the interesting phenomenon that arises due to a detail analysis of the dielectric properties of such composite. Beside detail analyzing, finding the way of enhancing the nonlinear optical response also got great attention. The presence of interfacial layer in the metal/dielectric composite is one way for enhancing the nonlinear optical response of the composite.

The nonlinear optical properties effect of the presence of single interfacial layer in a metal/dielectric composite has been studied by different researchers. All results shows that under proper condition the presence of interfacial layer increases the nonlinear optical properties of the composite [6, 7].

Recently, we studied the way of enhancing the enhancement factor of local field, and increasing the input domain threshold of the optical induced bistability of a spherical metal/dielectric composites within a linear host matrixes. By introducing double interfacial layer, the local field enhancement factor of a nanoparticle with dielectric core shows two maxima at two different frequencies and increases with increasing metallic fraction and metallic property of the second interfacial layer. Not only that, we also observed an increasing of the input domain threshold of the optical induced bistability for metallic property of the second interfacial layer [8].

The important aspect of this work deals with the effective nonlinear optical properties of composite media in which identical nonlinear nanospheres having double interfacial layer are randomly embedded in the linear host medium. In the metal/dielectric composite each nanosphers are consisting of dielectric core within a metal shell in a linear dielectric host matrix. Interfacial layers introduced between the dielectric core and the metallic shell and between the metallic shell and 
the linear dielectric host matrix. By considering double interfacial we study the effective third-order nonlinear susceptibility, refractive index, and absorption coefficient in a weakly-nonlinear case. Our results shows that large enhancement of optical nonlinear susceptibility, refractive index, and absorption can be achieved by varying the two interfacial factors at the plasma frequency.

The rest part of paper is structured as follows: Section 2, deals with the effects of double interfacial layers on optical nonlinear susceptibility, refraction index, and absorption coefficient of a metal/dielectric composite. In Section 3, we study numerically the effect of double interfacial layers on the optical nonlinear properties. Section 4 deals with summary and conclusion.

\section{Effects of double interfacial layers on nonlinear optical response, refraction index, and absorption coefficient of a metal/dielectric composite}

Let us consider a metal/dielectric composite randomly embedded in the linear host medium of dielectric function $\varepsilon_{h}$. Each nanospheres in the metal/dielectric composite are made up of a spherical shaped dielectric core of dielectric function $\varepsilon_{d}$, having an interfacial layer of dielectric function $\varepsilon_{s 1}$ within a metallic shell of dielectric function $\varepsilon_{m}$ that enclosed by another interfacial layer with a dielectric function $\varepsilon_{s 2}$ as shown fig.1.

We consider a nonlinear dielectric function of the core of nanosphere of the form;

$$
\varepsilon_{d}=\varepsilon_{d}^{(0)}+\chi_{d}\left|\mathbf{E}_{d}\right|^{2}
$$

that satisfies the displacement-electric field relation

$$
\mathbf{D}=\varepsilon_{d} \mathbf{E}_{d}=\varepsilon_{d}^{(0)} \mathbf{E}_{d}+\chi_{d}\left|\mathbf{E}_{d}\right|^{2} \mathbf{E}_{d}
$$

here $\mathbf{E}_{d}$ is the electric field inside the dielectric core of the nanosphere, and $\varepsilon_{d}^{(0)}$ and $\chi_{d}$ are linear dielectric function and cubic nonlinear susceptibility, respectively.

The electric field inside the dielectric core, that depends on the dielectric nature, is created because of the potential difference that produced by the external electric field applied to the nanosphere. We also assumed that $\chi_{d}\left|\mathbf{E}_{d}\right|^{2} \ll \varepsilon_{d}$.

In general, for finite $\omega, \mathbf{D}$ and $\mathbf{E}$ satisfies the Maxwell equations $\nabla \times \mathbf{E}=i \omega \mathbf{B}$ and $\nabla \times \mathbf{D}=0$ with $\mathbf{B}$ being the magnetic induction. However, for much less sizes of the nanosphere, compared to the wavelength of the incident light one can neglect the induction term $i \omega \mathbf{B}$ in Faradays law. So that, we can use a quasi-static approximation that satisfies the conditions $\nabla \times \mathbf{E}=0$ and $\nabla \times \mathbf{D}=0$. As a result we can get the simpler case that the metal/dielectric composites are immersed in a uniform but time-dependent field. Then using the Laplace equation, the potential distribution of the nanosphere can be written as follows:

$$
\begin{array}{cc}
\Phi_{d}=-\mathbf{E}_{h} A r \cos \theta, & r \leq r_{1} \\
\Phi_{s 1}=-\mathbf{E}_{h}\left(B r-\frac{C}{r^{2}}\right) \cos \theta, & r_{1} \leq r \leq r_{1}+t \\
\Phi_{m}=-\mathbf{E}_{h}\left(D r-\frac{E}{r^{2}}\right) \cos \theta, & r_{1}+t \leq r \leq r_{2} \\
\Phi_{s 2}=-\mathbf{E}_{h}\left(F r-\frac{G}{r^{2}}\right) \cos \theta, & r_{2} \leq r \leq r_{2}+t \\
\Phi_{h}=-\mathbf{E}_{h}\left(r-\frac{H}{r^{2}}\right) \cos \theta, & r>r_{2}+t
\end{array}
$$

where, $\Phi_{d}, \Phi_{s 1}, \Phi_{m}$, and $\Phi_{s 2}$ are potentials of the dielectric core, the first interfacial layer, the metal, the second interfacial layer, and the linear dielectric host matrix with respective radiuses of $r_{1}, r_{1}+t, r_{2}, r_{2}+t$ respectively. $\Phi_{h}, \mathbf{E}_{h}$ are the potential of the host dielectric and applied filed and $r$ and $\theta$ are the spherical coordinates respectively. Since we considered a quasistatic approximation, the electric potentials at the interfaces are continuous and there are no free charges at the interface surfaces, the normal component of the displacement vector is continuous across the interface surface. So the values of A, B, $\mathrm{C}, \mathrm{D}, \mathrm{E}, \mathrm{F}, \mathrm{G}$, and $\mathrm{H}$ can be obtained using the potentials and displacements vectors conditions of continuity. The expression for the induced dipole moment of the polarization $\mathrm{H}$ can be written as,

$$
\begin{gathered}
H=\alpha r_{2}^{3} \\
\alpha=1-\frac{3 \varepsilon_{h}\left(\left(\varepsilon_{d}+\frac{2 I_{1}}{r_{1}}\right)+\varepsilon_{m}\left(\frac{3}{p}-1\right)\right)}{2 \Delta}
\end{gathered}
$$




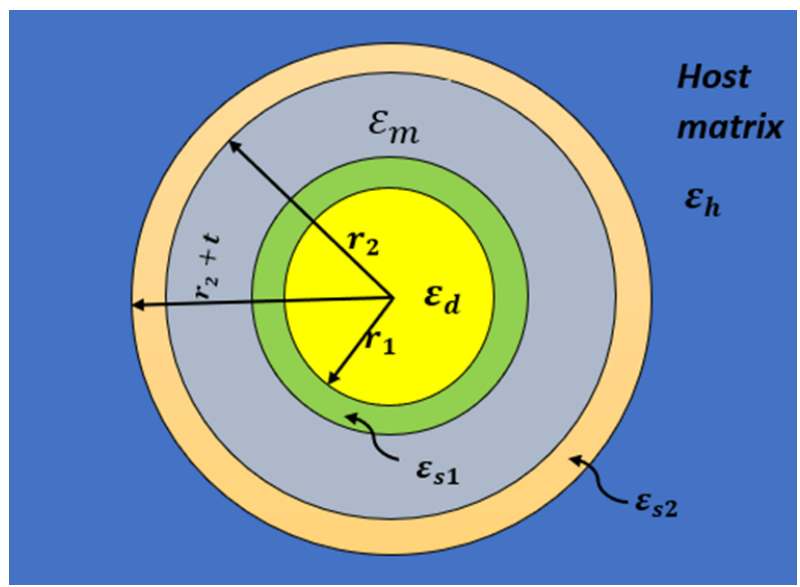

Figure 1. Metal/dielectric composite having double interfacial layers in a host matrix.

where $\Delta=\varepsilon_{m}^{2}+Q \varepsilon_{m}+\left(\varepsilon_{d}+\frac{2 I_{1}}{r_{1}}\right)\left(\varepsilon_{h}+\frac{I_{2}}{r_{2}}\right)$ with $Q=\left(\frac{3}{p}-1\right)\left(\varepsilon_{h}+\frac{I_{2}}{r_{2}}\right)+\left(\frac{3}{2 p}-1\right)\left(\varepsilon_{d}+\frac{2 I_{1}}{r_{1}}\right)$ and $p=\left(1-\left(\frac{r_{1}}{r_{2}}\right)^{2}\right)$ be the metal fraction in the inclusion. $I_{1}$ and $I_{2}$ are the interfacial layer factors lies between the dielectric core and the metal and the metal and the dielectric host matrix respectively.

The interfacial layers have the characteristic in the limit, $t \rightarrow 0$ (zero thicknesses), in the limit $\varepsilon_{s 1}, \varepsilon_{s 2} \rightarrow \infty$ with significant value of $\varepsilon_{s 1} t, \varepsilon_{s 2} t$ and defined as [9]

$$
I_{1}=\lim _{\varepsilon_{s 1} \rightarrow \infty, t \rightarrow 0} \varepsilon_{s 1} t, \quad I_{2}=\lim _{\varepsilon_{s 2} \rightarrow \infty, t \rightarrow 0} \varepsilon_{s 2} t
$$

Depending on the nature of the interfacial layer, the values of $I_{1}$ and $I_{2}$ could be zero or positive (negative). The values are zero when there is no interfacial layer. A positive value of the interfacial layer shows dielectric behavior of the interfacial layer and metal-like behavior of the interfacial layer for negative value [7] .

Since the nonlinear inclusion dielectric function is the form $\varepsilon_{d}=\varepsilon_{d}^{(0)}+\chi_{d}\left|\mathbf{E}_{d}\right|^{2}$, then, for a weak nonlinearity one can use Taylor expansion method for eq.9;

$$
\alpha=1-\frac{3 \varepsilon_{h} \rho}{2 \Lambda}\left(1-\frac{\kappa \chi_{d}\left|\mathbf{E}_{d}\right|^{2}}{\Lambda}\right)\left(1+\frac{\chi_{d}\left|\mathbf{E}_{d}\right|^{2}}{\rho}\right)
$$

$\left.\rho=\left(\varepsilon_{d}^{(0)}+\frac{2 I_{1}}{r_{1}}\right)+\varepsilon_{m}\left(\frac{3}{p}-1\right)\right), \kappa=\left(\frac{3}{2 p}-1\right) \varepsilon_{m}+\varepsilon_{h}+\frac{I_{2}}{r_{2}}, \Lambda=\varepsilon_{m}^{2}+Q^{\prime} \varepsilon_{m}+\left(\varepsilon_{d}^{(0)}+\frac{2 I_{1}}{r_{1}}\right)\left(\varepsilon_{h}+\frac{I_{2}}{r_{2}}\right), Q^{\prime}=\left(\frac{3}{p}-1\right)\left(\varepsilon_{h}+\right.$ $\left.\frac{I_{2}}{r_{2}}\right)+\left(\frac{3}{2 p}-1\right)\left(\varepsilon_{d}^{(0)}+\frac{2 I_{1}}{r_{1}}\right)$

For a weak nonlinearity it is convenient to consider the first order of $\chi_{d}$. Then we obtain an expression of $\alpha$ in the form

$$
\alpha=\alpha^{\prime}+\alpha^{\prime \prime} \chi_{d}\left|\mathbf{E}_{d}\right|^{2}
$$

with

$$
\alpha^{\prime}=1-\frac{3 \varepsilon_{h}\left(\left(\varepsilon_{d}^{(0)}+\frac{2 I_{1}}{r_{1}}\right)+\varepsilon_{m}\left(\frac{3}{p}-1\right)\right)}{2 \Lambda}
$$

and

$$
\alpha^{\prime \prime}=(1-p) 3 \varepsilon_{h}\left(\frac{3 \varepsilon_{m}}{2 p \Lambda}\right)^{2}
$$

The effective optical response according to the Clausius Mosotti relation, can be expressed as

$$
\varepsilon_{e}=\varepsilon_{h}[1+3 f \alpha]
$$

where $f=\frac{4 \pi}{3} N r_{2}^{2}$ is the volume fraction of metall/dielectric nanoparticles and $N$ is the number of spherical metall/dielectric particles per unit volume.

In principle, the local field $\mathbf{E}_{h}$ is uniform in the dielectric core of the nanosphere. And can be solved exactly by calculating the constant $A$ using the boundary conditions. From the the electric potential within dielectric core one can obtain the local field inside the dielectric core of the nanosphere and is given by

$$
\mathbf{E}_{d}=\frac{9 \varepsilon_{m} \varepsilon_{h}}{2 p \Delta} \mathbf{E}_{h}
$$


For a weak incident field $\chi|\mathbf{E}|^{2} \ll \varepsilon_{d 0}$ the contribution of the nonlinear kerr coefficient part of the inclusion dielectric becomes negligible. The local field inside the dielectric core becomes independent of $\chi|\mathbf{E}|^{2}$ and one can study the enhancement factor. The find enhancement factor of the optical-nonlinear response it is enough to calculate the local field in the core. This is because of restriction of nonlinearity of the nanosphere only in the core region. Due to the dielectric properties of the composite and plasma frequency, the enhancement factor of the local field in a metal spherical inclusion with a dielectric core in a linear host matrix has two maxima at two different frequencies. The enhancement factor maxima peaks increases in the presence of metallic property of dielectric and further increases in the presence of double interfacial layers of metallic properties. This is because of increasing plasm frequency in the presence of metallic interfacial layer [8].

However, the value of $\varepsilon_{d}$ inside $\Delta$ depends on $\mathbf{E}_{d}$. For a weak nonlinearity, after iterating, it is convenient keeping terms of only first order in $\chi_{d}$. Then the local field inside the core of the nanosphere becomes

$$
\mathbf{E}_{d}=\frac{9 \varepsilon_{m} \varepsilon_{h}}{2 p \Lambda} \mathbf{E}_{h}-\frac{9 \varepsilon_{m} \varepsilon_{h}}{2 p \Lambda^{2}} \zeta\left|\frac{9 \varepsilon_{m} \varepsilon_{h}}{2 p \Lambda}\right|^{2} \chi_{d}\left|\mathbf{E}_{h}\right|^{2} \mathbf{E}_{h}
$$

where $\zeta=\left(\frac{3}{2 p}-1\right) \varepsilon_{m}+\left(\varepsilon_{h}+\frac{I_{2}}{r_{2}}\right)$

Moreover, by considering the ClausiusMosotti relation, in which we assume that the inclusions are not too densely packed and only dipolar interaction between the inclusions are taken into account, we obtained the macroscopic effective linear dielectric function $\varepsilon_{e}$ and third-order nonlinear susceptibility $\chi_{e}$ and introduced as follows:

$$
\varepsilon_{e}=\varepsilon_{e}^{(0)}+\chi_{e}\left|\mathbf{E}_{h}\right|^{2}
$$

with

and

$$
\varepsilon_{e}^{(0)}=\varepsilon_{h}+3 \varepsilon_{h} f \alpha^{\prime}
$$

$$
\chi_{e}=3 \varepsilon_{h} f \alpha^{\prime \prime} \chi_{d}\left|\frac{9 \varepsilon_{h} \varepsilon_{m}}{2 p \Lambda}\right|^{2}
$$

Furthermore, we consider Drude form for the metal dielectric function in the inclusion and is given by

$$
\varepsilon_{m}=\varepsilon_{\infty}-\frac{1}{Z(Z+i \gamma)}
$$

with dimensionless frequencies

$$
Z=\omega / \omega_{p}, \quad \gamma=\nu / \omega_{p}
$$

where $\omega, \omega_{p}$ and $\nu$ are the incident radiation frequency, the metal shell frequency, and the electron collision frequency respectively.

The above equation can be written in the form of real and imaginary parts as

$$
\varepsilon_{m}=\varepsilon_{m}^{\prime}+i \varepsilon_{m}^{\prime \prime}
$$

where $\varepsilon_{m}^{\prime}=\varepsilon_{\infty}-\frac{1}{\left(Z^{2}+\gamma^{2}\right)}, \varepsilon_{m}^{\prime \prime}=\frac{\gamma}{Z\left(Z^{2}+\gamma^{2}\right)}$ and $\varepsilon_{\infty}$ is the high frequency dielectric constant.

By considering only the metallic dielectric complex nature, we can rewrite the effective dielectric function $\varepsilon_{e}$ in terms of real and imaginary part of the form

$$
\varepsilon_{e}=\epsilon_{1}+\epsilon_{2} i
$$

with real part

and imaginary part

$$
\epsilon_{1}=\varepsilon_{h}+3 \varepsilon_{h} f R e\left\{\alpha^{\prime}\right\}+3 \varepsilon_{h} f R e\left\{\alpha^{\prime \prime}\right\} \chi_{d}\left|\frac{9 \varepsilon_{h} \varepsilon_{m}}{2 \Lambda}\right|^{2}
$$

$$
\epsilon_{2}=3 \varepsilon_{h} f \operatorname{Im}\left\{\alpha^{\prime}\right\}+3 \varepsilon_{h} f \operatorname{Im}\left\{\alpha^{\prime \prime}\right\} \chi_{d}\left|\frac{9 \varepsilon_{h} \varepsilon_{m}}{2 p \Lambda}\right|^{2}
$$

The refractive index and the absorption coefficient are related with the dielectric function by the following relation

$$
n+i k=\sqrt{\varepsilon_{e}}
$$

where $n$ is the refractive index of the medium and $k$ is the absorption coefficient [10]. Using equation (eq. 24) we obtain the refractive index $n$ and absorption coefficients of the form

and

$$
n=\sqrt{\frac{1}{2}\left(\epsilon_{1}+\sqrt{\left(\epsilon_{1}^{2}+\epsilon_{2}^{2}\right)}\right)}
$$

$$
k=\sqrt{\frac{1}{2}\left(-\epsilon_{1}+\sqrt{\left(\epsilon_{1}^{2}+\epsilon_{2}^{2}\right)}\right)}
$$

In the next section we will discuses the nonlinear susceptibility, the refractive indexes, and absorption coefficients with different controlling parameters by taking the data numerically. 


\section{Numerical Calculation and Discussion}

In this section we formulated in detail the third-order nonlinear susceptibility, the refractive index and absorption coefficient in terms of the the interfacial factor $I_{1}$, the second interfacial layer $r_{2}$, and the incident electric field frequency. For the whole process, we take $\varepsilon_{\infty}=4.5, \varepsilon_{d}=6, \varepsilon_{h}=2.25, \omega_{p}=1.46 \times 10^{16}$ and $\nu=1.68 \times 10^{14},\left|\mathbf{E}_{h}\right|=0.05$, and $\chi_{d}=1$ with arbitrary units. We plot all the refractive indexes and absorption coefficients by considering the first order of optical nonlinear susceptibility of the dielectric core.
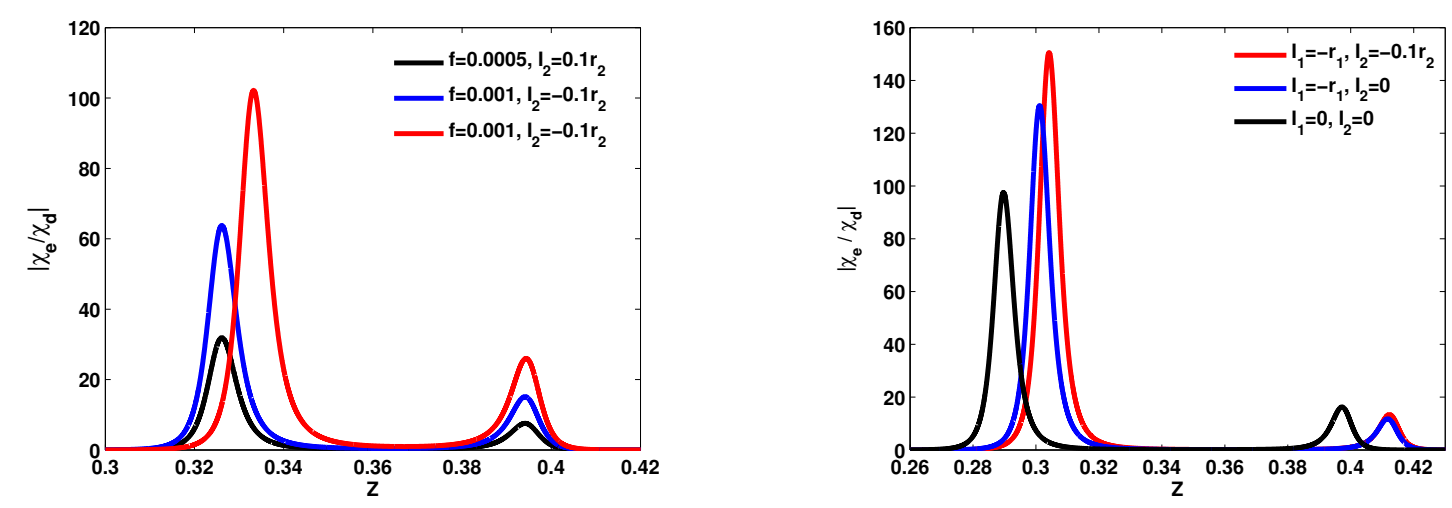

Figure 2. (a) (Color online) $\left|\frac{\chi_{e}}{\chi_{d}}\right|$ versus $Z$ of the effective composite with $f=0.001$ and $I_{2}=-0.1 r_{2}$ (red line), $f=0.001$ and $I_{2}=0.1 r_{2}$ (blue line) and $f=0.0005$ and $I_{2}=0.1 r_{2}$ (black line). Other parameter $p=0.99$. (b) $\left|\frac{\chi_{e}}{\chi_{d}}\right|$ versus $Z$ of the effective composite without interfacial layer (black line), with single interfacial layer of $I=-1 r_{1}$ (blue line), and double interfacial layer with $I_{1}=-1 r_{1}$ and $I_{2}=0.1 r_{2}($ red line).The value $p=0.9$

In fig. 2a we plot the magnitude of the ratio of third-order nonlinear susceptibility of the effective medium with thirdorder nonlinear susceptibility of the dielectric core with different second interfacial factor of $I_{2}=0.1 r_{2}$ (blue line) and $I_{2}=-0.1 r_{2}$ (red line) at plasma frequency. Plasma frequency is the highest frequency which electron in the metal/dielectric composite could be able to respond with, being equal to say highest for plasma too (Ions mass is much larger than electron). Thus, when electromagnetic wave with larger frequency goes through the plasma, no particle has enough time to respond, so no considerable change will happen to the incident wave. Regardless of the character of the first interfacial layer, the magnitude of the ratio of third-order nonlinear susceptibility of the effective medium with the dielectric core susceptibility increase when the second interfacial layer change from dielectric property to metallic property. Moreover, the magnitude of the ratio of third-order nonlinear susceptibility of the effective medium with the dielectric core susceptibility increase with increasing volume fraction of metall/dielectric nanoparticles ( blue color and red color in fig. 2a). This is because of the fact that within the two interfacial layer the metallic property becomes more dominant with increasing metallic friction.

Fig. $2 b$, we compare the magnitude of the ratio of third-order nonlinear susceptibility of the effective medium with the dielectric core susceptibility for a composite without interfacial layer, having single metallic interfacial layer, and with double interfacial layer having inner and outer of metallic properties of interfacial layers of the same composite. Accordingly, in the presence of double interfacial layers the ratio $\left|\chi_{e} / \chi_{d}\right|$ increases. However, the ratio $\left|\chi_{e} / \chi_{d}\right|$ decreases with double interfacial layers with dielectric property of the first and second interfacial layer. So, one can control the nonlinear response by adjusting the natures of the two interfacial layers.

We also numerically calculate and plot the refractive index of the composite having different volume fraction of metall/dielectric nanoparticles and second interfacial factor as a function of frequency at plasma frequency as show in fig. $3 \mathrm{a}$. Our result shows two maxima peaks that occurred at different frequencies. With increasing the volume fraction of metall/dielectric nanoparticles both the first and second maxima of the refractive index increase. With changing the second interfacial layer factor of the interfacial layer from dielectric-like to metal-like property, the refractive index of the effective composite enhanced. In comparison with first maxima, the second maxima peak of the refractive index is highly enhanced for metallic property of the second interfacial layer.

Figure $3 \mathrm{~b}$ shows the refractive index in the absence of interfacial layer (black line), with single interfacial layer (blue line) and double interfacial layer (red line) at plasma frequency. Our finding shows that because of double interfacial layer the both the first and second maxima peak increase for metallic property of the first interfacial layer and the second interfacial layer. On the other hand, with dielectric property of the first and second interfacial factor, the maxima peaks decreases. We noticed that the presence of second interfacial layer plays a contractive role on adjusting the refractive index of the effective composite.

The absorption coefficients are plotted as a function of frequency for different volume fraction of metall/dielectric nanoparticles and second interfacial factors at plasma frequency as shown in fig. 4a. The effective medium composite absorption reach a maximum at two different frequencies. For increasing volume fraction of metall/dielectric nanoparticles, both the 

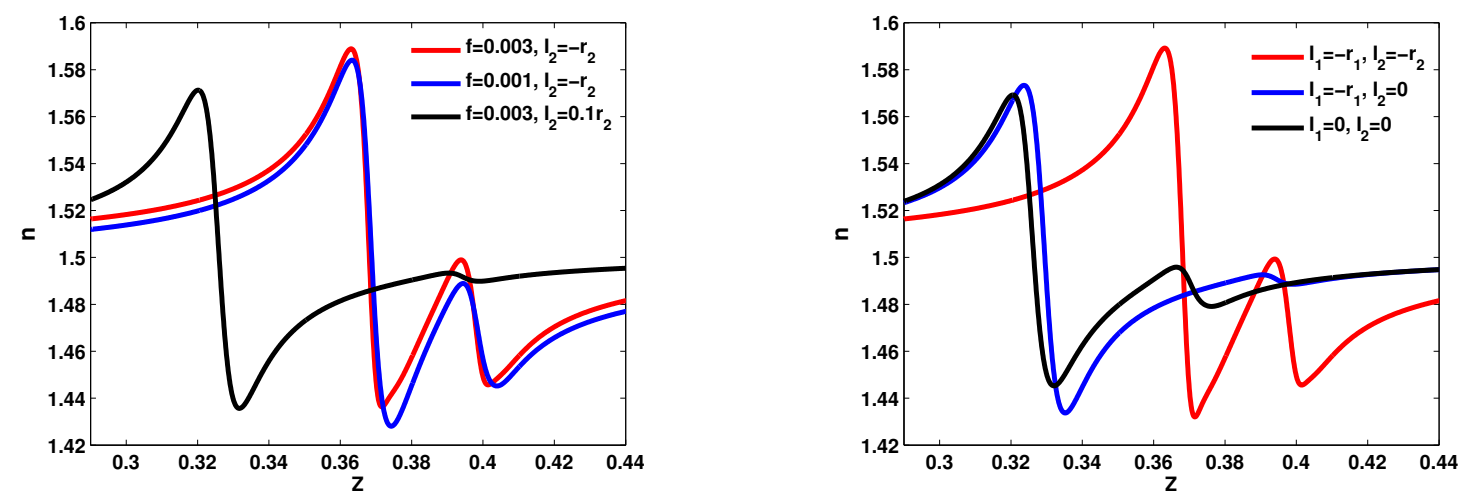

Figure 3. (a) (Color online) (a) The refractive index of the effective composite versus z for $f=0.003$ and $I_{2}=-1 r_{2}$ (red line), $f=0.001$ and $I_{2}=-1 r_{2}$ (blue line) and $f=0.003$ and $I_{2}=0.1 r_{2}$ (black line) in linear host matrix. In both plots of $I_{1}=-1 r_{1}$, (b)The refractive index of the effective composite versus $\mathrm{z}$ for without interfacial layer (black line), with single interfacial layer of $I=-1 r_{1}$ (blue line) and double interfacial layer of $I_{1}=-1 r_{2}$ (black line) and $I_{2}=-1 r_{2}$ (red line) in linear host matrix with $f=0.003$.
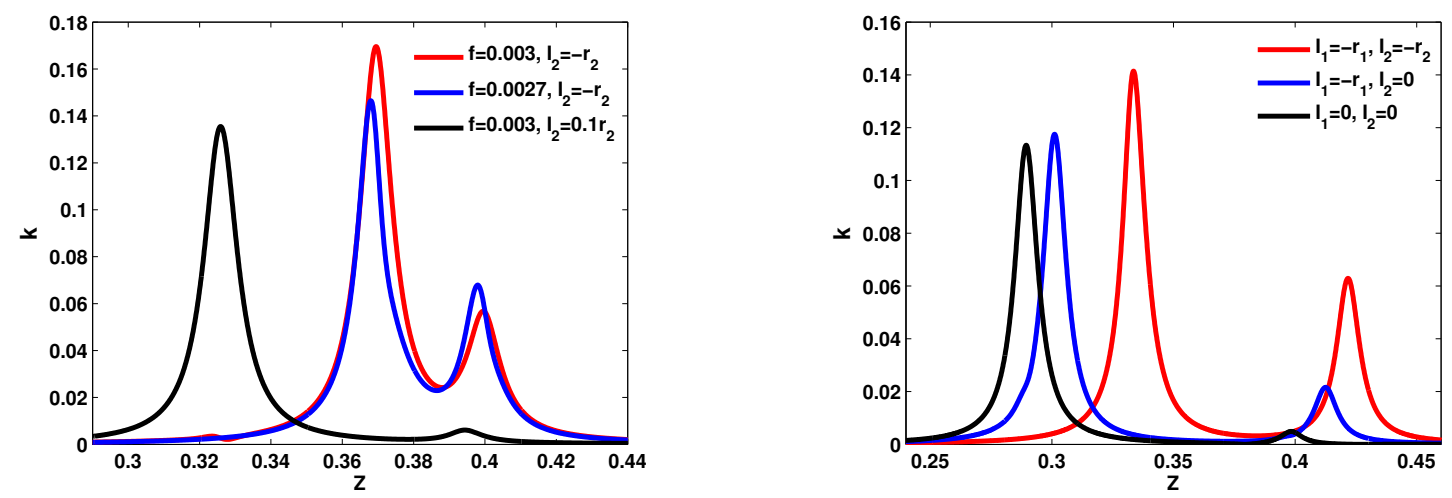

Figure 4. (a) (Color online) (a) The absorption coefficient of the effective composite versus z for $f=0.003$ and $I_{2}=-1 r_{2}$ (red line), $f=0.0027$ and $I_{2}=-1 r_{2}$ (blue line) and $f=0.003$ and $I_{2}=0.1 r_{2}$ (black line) in linear host matrix. In both plots of $I_{1}=-1 r_{1}$ and $p=0.99$, (b)The refractive index of the effective composite versus $\mathrm{z}$ for without interfacial layer (black line), with single interfacial layer of $I=-1 r_{1}$ (blue line) and double interfacial layer of $I_{1}=-1 r_{2}$ and $I_{2}=-1 r_{2}$ (red line) in linear host matrix with $f=0.003$ and $p=0.9$.

first and the second maxima peaks increases (compare the red and blue colors in fig. 4a). Moreover, with increasing metallic like property of the second interfacial layer the two maxima peaks increases. However, with increasing dielectric property of the second interfacial layer, both the first and the second maxima peaks decreases with a high rate of decreasing the second maxima peak.

In fig. $4 \mathrm{~b}$ we compare the absorption coefficient in the absence of interfacial layer (black line), with single interfacial layer (blue line) and double interfacial layer (red line). For metal like property of the first and second interfacial layers both the first and second maxima peaks are greater than that of a composite having single metallic like interfacial layer and a composite without interfacial layer. On the other hand, for dielectric like property of the first and second interfacial layers the maxima peaks are lowers than that of a composite having single dielectric like interfacial layer and a composite without interfacial layer. So, one can study other properties of the composite by altering the nature of the second interfacial layer.

In general, our results shows that the optical nonlinear third-order susceptibility, the refractive index and the absorption coefficients of a metal/dielectric composite increases for increasing volume fraction of metall/dielectric nanoparticles and increasing metallic property of the second interfacial layer So, depending on the nature of the second interfacial layer one can control the optical nonlinear third-order susceptibility, the refractive index and the absorption coefficients of a metal/dielectric composite.

\section{Summary and Conclusion}

In this work we studied theoretically and calculated numerically the effect of double interfacial layers of a metal/dielectric composite randomly embedded in the linear host medium. The metal/dielectric nanosphere is composed of dielectric core and metallic outer shell in which the interfacial layers lies between the dielectric core and the metal shell and between the metal shell and the host matrix. We considered the ClausiusMosotti approximation in which the inclusions are not too densely 
packed and only dipolar interaction between the inclusions are taken into account.

Depending on volume fraction of metall/dielectric nanoparticles and the interfacial factor of the second interfacial layer, the optical nonlinear third-order susceptibility, refractive index, and absorption coefficient are studied. Our result shows the best way of enhancing the effective nonlinear optical properties of such composite. According to our finding all the optical nonlinear third-order susceptibility, refractive index, and absorption coefficient shows two maxima peaks at two different resonant frequencies.

The maxima peaks of the optical nonlinear third-order susceptibility increases with increasing volume fraction of metall/dielectric nanoparticles. Not only the optical nonlinear third-order susceptibility, but the refractive indexes and absorption coefficients also increases with increasing volume fraction of metall/dielectric nanoparticles. Moreover, all the optical nonlinear third-order susceptibilities, refractive indexes, and absorption coefficients increases because of shifting the interfacial factor from dielectric to metallic properties.

In comparison with a composite without interfacial layer and a composite having single interfacial layer, the presence of the second interfacial layer, exhibit maximum peaks of the two maxima of the third-order susceptibility, refractive index, and absorption coefficient for metallic like property of the second interfacial layer.

In conclusion, this work proposed an alternative ways of enhancing the optical nonlinear third-order susceptibility, refractive index, and absorption coefficient by introducing additional interfacial layer in the nanosphere of metal/dielectric composite. It also helps for further study nonlinear optical properties of such composite.

\section{Acknowledgment}

The author would like to thank Dr. Sisay Shewamare for material support during the making of this research work.

\section{REFERENCES}

[1] E. Garmire, http://www.intechopen.com/books/nonlinearoptics/overview-of-nonlinear-optics.

[2] D. A. B. Miller, Proc. IEEE 97, 11661185 (2009)

[3] Robert W. Boyd Nonlinear optics, Academic Press (Elsevier Science, USA, 2003).

[4] D.J. Bergman, O. Levy and D. Stroud, Phys. Rev. B 44129 (1994).

[5] F. Brouers, J.P. Clerc and G. Giraud, Phys. Rev. B 445299 (1991).

[6] Huang Ji-Ping, Gao Lei, and Li Zhen-Ya, Commun. Theor. Phys. 36251 (2001).

[7] T. Pan, J. P. Huang, and Z. Y. Li, Physica B 301, 190195 (2001).

[8] Berhanu Aragie, Universal Journal of Physics and Application 13, 8-14 (2019)

[9] S. Torquato, M. D. Rintoul, Phys. Rev. Lett. 75, 4067 (1995).

[10] L.D. Landau and E.M. Liftshitz, Course of Theoretical Physics, Electrodynamics of Continuons Media, (2nd edition, Vol. 8, Pergamon Press (1984)). 